\title{
Energy Aware Routing Protocol for Energy Constrained Mobile Ad-hoc Networks
}

\author{
Nadeem Iqbal, Mohammad Shafie Bin Abd Latiff, Shafi'i Muhammad Abdulhamid \\ Universiti Teknologi Malaysia, Malaysia
}

\section{Article Info}

Article history:

Received Nov 27, 2017

Revised Dec 31, 2017

Accepted Jul 10, 2018

\section{Keyword:}

AODV

Energy-efficient

MANET

Power-aware metrics

Routing protocols

\begin{abstract}
Dynamic topology change and decentralized makes routing a challenging task in mobile ad hoc network. Energy efficient routing is the most challenging task in MANET due to limited energy of mobile nodes. Limited power of batteries typically use in MANET, and this is not easy to change or replace while running communication. Network disorder can occur for many factors but in middle of these factors deficiency of energy is the most significant one for causing broken links and early partition of the network. Evenly distribution of power between nodes could enhance the lifetime of the network, which leads to improving overall network transmission and minimizes the connection request. To discourse this issue, we propose an Energy Aware Routing Protocol (EARP) which considers node energy in route searching process and chooses nodes with higher energy levels. The EARP aim is to establish the shortest route from source to destination that contains energy efficient nodes. The performance of EARP is evaluated in terms of packet delivery ratio, network lifetime, end-to-end delay and throughput. Results of simulation done by using NS2 network simulator shows that EARP can achieve both high throughput and delivery ratio, whereas increase network lifetime and decreases end-to-end delay.
\end{abstract}

Copyright (c) 2018 Institute of Advanced Engineering and Science. All rights reserved.

Corresponding Author:

Nadeem Iqbal, Universiti Teknologi Malaysia, Malaysia.

Email: ndiqbal@gmail.com

\section{INTRODUCTION}

No fix host and router is providing an access point in mobile ad hoc network. Data packets are sent and received by portable users away from the access point by using wireless communication capability of mobile nodes through other neighbouring nodes building active networks [1], [2], [3]. Communication between mobile nodes is done by intermediate nodes directly or indirectly [4], [5], [6]. Due to secure deployments and non-restricted features of MANETs, it is very appropriate and standard for applications like military operations, emergencies and natural disasters [7], [8], [9].

Figure 1 demonstrates an instance of MANET. Mobile nodes affected mostly by exhausting battery power. MANET node depends on power or energy. Due to the limitation of energy resources and it is not possible to preserve energy for a longer time, transmitting or receiving may stop for the irregular period which leads to selfish behaviour of nodes [10]. Data forwarding in the route done with cooperative nodes which are active. A packet forwarding may stop by selfish nodes for saving their energy which causes inefficient data transmission [11], [12]. Enhancement of network lifetime can achieve through energy efficient operations. Consequently, energy efficiency is vital significance in the designing of routing algorithm for the applications in such networks, and streamlined procedures are acute to increase the network lifetime. 


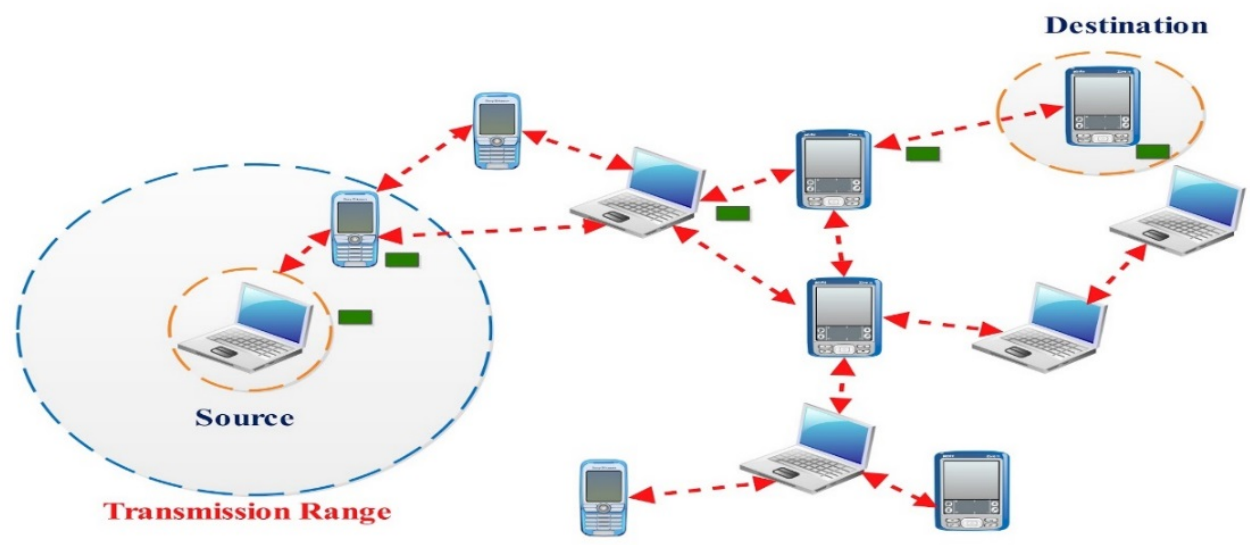

Figure 1. Mobile ad hoc network

Maximizing every node lifetime in the network which increases the overall life of network and minimizing total energy consumption of the route are essential features of Energy Efficient Routing Protocols. Maintaining the network functioning longer time as much as possible is the focal determination of Energy Efficient Routing Protocols [13], [14], [15]. Transmitting, receiving and sleeping states of nodes are energy consumption states in MANET. In comparison with sleep, state node consumes more energy when transmitting. When nodes are not transmitting or receiving any signal is called sleep state means nodes are idle. To achieve the longer network operational objective, node energy consumption should be reduced not only during the transmission but also during sleep states [16], [17]. Mobile nodes are battery-powered, due to this, battery power is a valuable source that has to be cautiously used by the nodes to evade an early network partition and hence the study and the operation of power-aware algorithms for MANET quite establishes a massive area for researchers.

The traditional routing schemes cannot be applied as they are unable to detect nodes' remaining energy and the cost associated with routing data packet. In recent past, some energy efficient schemes have shown a more attractive solution for node selfishness. However, the majority of the current energy efficient schemes shows limitations to deal with selfish nodes due to the method used and its consequences in the inaccurate making of the decision. Moreover, these protocols demonstrate outdated unrestrained learning of other nodes and continuously function to overhear which creates high control overhead in the network and consumes more energy.

\section{REVIEW OF EXISTING WORK}

Several energy-aware routing protocols have presented by researchers for the mobile ad-hoc network. Authors in [18] recommended AODV-EER (end-to-end reliable AODV) route reliability in AODV from the source node to destination node. This scheme selects a path to the destination with the minimum rate of packet dropping. If there is a breakage in the data path of the primary route, then a mechanism called backwards route entry initiates, and it increases the route stability by selecting the more stable path for sending data from source node to destination node. In the beginning it chooses a path with those nodes which have stable links as a substitute for hop-by-hop selection. Therefore, it degrades the link breakage probabilities and failure of the network. In addition to this, end-to-end node reliability is prioritized in the selection of primary routes with high consistency. It adds extra fields in the data packet (BRR) Backward_Reliable_Route, and the other one is (BID) Backward_ID.

Node address is kept by BID for standby path to the destination while BRR keeps tracks on route consistency to the destination node from the node of BID. In case of any route, breakage occurs between nodes, the secondary route is checked for substitution of the broken link. BID data is searched by that node in the event of no substitute route found. However, there is no solution for error in AODV-EER as it does not provide any technique to deal with notification of error from nodes. Moreover, reliable rates of hops are used, and it selects routes which are the more reliable rate, while computation of reliability rate consumes energy and it causes the source to destination delay when nodes are in high mobility. Furthermore, it does not provide any mechanism for tracking link breakage, and there is no update for link quality. For efficient route selection [19] proposed M-AODV it selects efficient route by using overhearing backup route. This scheme 
combines the characteristics of AODV-ABL and AR-AODV algorithm. Moreover, in the first phase, it checks for the alternative route in data packet forwarding if there is no alternative is there or alternative is already expired then it goes in the second phase by using AODV-ABL local route repair, or it uses an alternative route for it with one-hop neighbour. Furthermore, overhead information is compared with the table of the main route, and it will be replaced with better route entry in the routing table. However, in route discovery process M-AODV uses the both AR-AODV and AODV-ABL schemes, and as a result, it adds the latency of both algorithms. Therefore, it increases packet delay and reduces overall network performance. Moreover, it saves both main route and alternative route information at the same time in the routing table. Therefore, a significant amount of memory, bandwidth, and energy is consumed due to routing table update which causes this scheme to become inefficient. Furthermore, due to change in network condition alternate route is not necessarily an optimal route; therefore it became obsolete as the discovery of alternate route is in the same interval of time as the original route was found during the same route discovery process.

A different route repair approach for the mobile ad-hoc network discussed in [20]. They proposed multicast in AODV protocol with maximum energy local route repair (AODV-LRR), for link breakage it uses local route repair technique. Also, it repairs the route in case of energy depletion of the node from the same point where the breakage occurs deprived of informing sender node. It follows two phases, firstly it chooses the path and delivers the data packets to the right node in the destination. The intermediate node selects sender node by higher energy levels of neighbour node among other nodes in the network. Therefore, it always chooses the nodes which have higher energy levels in route selection. In the second phase if any link breakage due to node mobility or energy reduction it reconstructs route by using local route repair technique from the point where it breaks. However, there is no provision for multiple link breakage at the same point in AODV-LRR. Moreover, it compares route energy levels for better route selection which devours energy and creates delays. In addition to this, there is no mechanism to send route breakage information to sender node which introduces packet error.

In [21] Enhanced Receiver Based AODV (ERB_AODV) proposed. This scheme works in two different stages discovery and maintenance stage. In the first stage, sender node checks its routing table for existing route to the destination if no route is available then it broadcasts the control packet towards sender node in search of a path to the receiver node. Moreover, it minimizes the control overhead by using broadcast control packet in route search in maintenance stage which reduces overhead when nodes are in high mobility and load. Furthermore, it uses the receiver control agent, so that receiver node controls communication status in the maintenance stage. However, ERB_AODV route discovery process takes longer time which creates network overhead. In addition to this, there is no consideration for quality of a link while selecting an intermediate node and due to variable link nature breaks the link significantly which increases retransmission of data packet, and it consumes more energy. Another scheme Enhanced AODVsuggested in [22]. It calculates in the first step the total nodes and actual power of those nodes in the route, later on, if any path or link failure is found it checks for the number of hops data so far travelled and remaining number of hops to reach the destination. Also, an alternate data path utilized for improving data delivery ratio. However, there is no mechanism in Enhanced AODV to deal with error as there is no reporting for nodes in error also it calculates hops and node energy in path selection and in high mobility network model it creates the delay.

Better route selection and route repair is proposed in [23] and named it B-AODV. Reverse route request $\mathrm{B}-\mathrm{RREQ}$ is using in this scheme in case of RREP for searching routes. B-RREQ is using as a control message for reverse route establishment. At first occurrence of B-RREQ node in source saves next hop information and data transfer starts. However, in B-RREQ routing table is updated for each B-RREQ and RREQ information which requires more energy and produces network delay. Moreover, it does not share link breakage information from sender node which creates packet transmission error. To reduce link failure, a method is suggested in [24] to repair a link through preventative local route repair in basic AODV scheme. Modified AODV compares node residual energy by two different values of threshold which provides information about link failure and type of operation. After checking the particular level of node residual energy, it will provide information of insufficient node energy to stop sending data packets from the specified node by modifying route request and route reply data packets. However, this scheme performs an additional procedure of node remaining energy comparison with two different threshold values which produces the delay in case of nodes are in high mobility. Moreover, there is no mechanism to deal with node error recommendations.

Limitation of Reverse AODV (R-AODV) scheme is considered in [25] and proposed (SEARAODV) Stability and Energy Aware Reverse AODV scheme. It considers node reliability factor which contains energy and route stability as a metric. It selects a primary path for the route with higher reliability factor for data sending, and it chooses the secondary route by next high-reliability factor. Also, it broadcast R-RREQ and RREQ packets to source node as well as destination node to reduce route maintenance process. However, this scheme uses R-RREQ and RREQ broadcast which produces more overhead and energy 
consumption which creates the delay in data transmission. Moreover, the weaker signal of intermediate nodes is checked for each time to verify path availability in the routing table. Furthermore, there is no method provided to deal with transient link failures due to nodes congestion and other causes of link failure notifications. Table 1 deliberates the summary and provides a comparison of discussed routing schemes. Associated parameters like detection mechanism of path breakage, maintenance of route, frequency to discover route, overheads of routing and stability of routes.

Table 1. Comparison and Summary of Reporting and link Failure Schemes

\begin{tabular}{|c|c|c|c|c|c|c|}
\hline Schemes & Methodology & $\begin{array}{c}\text { Detection } \\
\text { Mechanism }\end{array}$ & $\begin{array}{c}\text { Route } \\
\text { Maintenance }\end{array}$ & $\begin{array}{c}\text { Route } \\
\text { Discovery } \\
\text { Frequency } \\
\end{array}$ & $\begin{array}{c}\text { Route } \\
\text { Stability }\end{array}$ & $\begin{array}{c}\text { Routing } \\
\text { Overheads }\end{array}$ \\
\hline $\begin{array}{l}\text { M-AODV[19] } \\
2016\end{array}$ & Backup route & Hello-based & Conventional & Low & Medium & Moderate \\
\hline $\begin{array}{l}\text { ERB-AODV[21] } \\
2015\end{array}$ & Local repair & Hello-based & Conventional & High & Low & Moderate \\
\hline $\begin{array}{l}\text { SEAR-AODV[25] } \\
2015\end{array}$ & Backup route & $\begin{array}{c}\text { Link Layer } \\
\text { Based }\end{array}$ & Conventional & High & Low & High \\
\hline $\begin{array}{c}\text { AODV-EER [18] } \\
2014\end{array}$ & Backup route & $\begin{array}{l}\text { Link Layer } \\
\text { Based }\end{array}$ & Conventional & High & Low & High \\
\hline $\begin{array}{c}\text { AODV-LRR[20] } \\
2014\end{array}$ & Local repair & Hello-based & Conventional & High & Low & High \\
\hline $\begin{array}{c}\text { Modified AODV[24] } \\
2014\end{array}$ & Local repair & Hello-based & Conventional & High & Low & Moderate \\
\hline $\begin{array}{c}\text { Enhanced AODV[22] } \\
2013\end{array}$ & Backup route & Hello-based & Improved & Low & Medium & Moderate \\
\hline $\begin{array}{l}\text { B-AODV[23] } \\
2013 \\
\end{array}$ & Backup route & Hello-based & Conventional & High & Low & High \\
\hline
\end{tabular}

Locally route repair method is used to repair link breakage in mostly proposed routing schemes. However, traditional route maintenance mechanism which produces more and more route discoveries. Moreover, to detect link breakage link-layer base or Hello-based method is considered. Furthermore, default Hello-based method deliberated in most of the on-demand routing schemes which are more susceptible for notifications of link failure and continuously states about broken link in the network without considering disruption of transmission due to link congestion. To propose a new energy-aware routing protocol is the main contribution of this paper that incorporates with energy aware concept in routing protocol design which prolongs network lifetime and provides reliable data delivery from source to destination. The suggested protocol does not use more constraints and utilizes fewer resources, due to this it is more appropriate for resource constraints network environments. These features make Energy Aware Routing Protocol (EARP) a suitable choice for application in MANET in comparison with existing schemes.

\section{THE PROPOSED EARP SCHEME}

This section illustrates the details of proposed Energy Aware Routing Protocol (EARP). Before discussing design facts of EARP, certain assumptions used in the protocol design elaborated first.

\subsection{Assumptions}

Energy Aware Routing Protocol (EARP) developed with following fundamental assumptions:

a. Nodes are not added or removed from the network after initial deployment.

b. All nodes use the shared bidirectional wireless channel for data communication.

c. Same signal attenuation is used for communication between two nodes.

d. All nodes distribute arbitrarily position, movement and initial energy.

e. Link layer of the node is used to read energy information of that node through the physical interface, and it is capable of response network layer.

\subsection{Research Method}

The design of EARP contributes energy balancing between nodes, energy efficient route selection, node energy monitor and efficient route maintenance. EARP uses those mobile nodes in route selection which have higher energy levels, and it ensures that the selected route from source to the destination node is the shortest route for data transfer. A Combined Routing Function (CRF) uses Hop counts and node energy 
as a weight which implemented as a metric. Node remaining energy and Hop counts are sum up and considered as a weight to find out the CRF cost as illustrated in Equation 1.

$$
\text { Combined Routing Function }=\lambda \times \text { Node energy }+\theta \times \text { Hop count }
$$

Node energy and hop count is denoted by weights $\lambda$ and $\theta$ while the sum of these weights is equal to one. $(\lambda+\theta=1)$. Initially, an equal proportion is given to weights $\lambda$ and $\theta$ to have the same influence on path selection, but they can change as per requirements of the application. The node chosen in routing path which enhances combine routing function and this node shows perfect nominee for meeting the cohesive set of requirements. In node selection process only those node will be chosen whose residual energy is more than the fixed value of the threshold. Any intermediate node which receives route request packet due to source node broadcast it adds information of the number of hops till now traversed and its remaining energy in the header of the packet. Route request packet will discard if node energy value is smaller than the fixed threshold. At the destination, after receiving route request packet, it is added with hops field and send it back to the source. This process recaps until source receives the route reply packet. Multiple route replay packets from different paths will receive at the source node. The final decision made by the source node on routing cost of all routes.

\subsubsection{Route Setup}

Energy Aware Routing Protocol (EARP) customizes the control packets RREQ and RREP of AODV routing protocol for selection of shortest as well as energy efficient route for sending data from source to destination. Nodes with higher residual energy levels from fix threshold value will be selected for the route the data packet. Moreover, by choosing the shortest path with higher energy level nodes allows EARP to utilize few nodes in data transmission which minimizes overall energy consumption. For illustration, only those nodes added by upstream nodes which have higher energy levels. Consequently, in case of lack of energy level of a specific node will ignore RREQ packet in route discovery process. A network scenario is demonstrated in Figure 2 (a), where node $\boldsymbol{a}$ is source node and $\boldsymbol{d}$ is destination node. Only nodes with higher energy levels are selected from the source node $\boldsymbol{a}$ to make energy efficient route towards destination node $\boldsymbol{d}$ for sending data. Initially, RREQ broadcasted to all nodes which are in the neighborhood of source node $\boldsymbol{a}$, later on, the energy levels of nodes compared with fix threshold value and if it is found less than RREQ packet will discard from that node. As node $\boldsymbol{j}$, moreover there will be no participation from that in data communication, otherwise RREQ packet will further forwarded to neighbour node and it marks a reserve route entry for the source node $\boldsymbol{a}$. This process repeats until the node in the destination will receive the RREQ packet. Node $\boldsymbol{d}$ unicast the RREP packet towards source node $\boldsymbol{a}$ with reverse route. Source node $\boldsymbol{a}$ selects route $\boldsymbol{a} \rightarrow \boldsymbol{b} \rightarrow \boldsymbol{c} \rightarrow \boldsymbol{d}$ by using EARP mechanism, as the chosen route is shortest as well as energy aware. Figure 3 represents the flowchart of proposed EARP scheme route discovery process.Moreover, an improved route request is denoted by I-RREQ and I-RREP represents improved route reply. Furthermore, enhanced elements in the detection of augmented route selection are displayed by dotted shaded boxes.

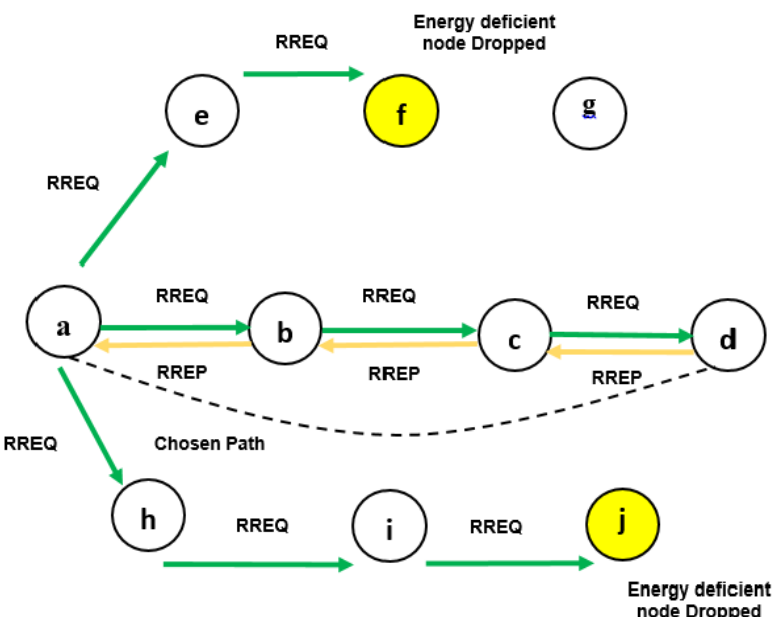

(a)

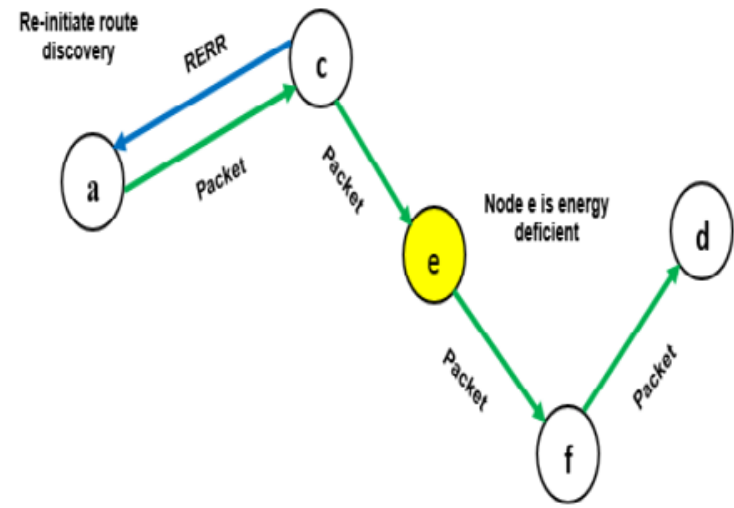

(b)

Figure 2. EARP route setup and route maintenance process 


\subsubsection{Route Maintenance}

EARP scheme uses an efficient Route Maintenance strategy that enhances the intermediate node role for power-aware route maintenance. Moreover, in case of any node energy is less than the specified threshold in an ongoing communication then EARP restarts route discovery phase and informs upstream node for new route by eliminating energy depletion node. The Figure 2(b) explains the situation where node creports the condition $\boldsymbol{R} . \boldsymbol{E}<\boldsymbol{T h}$ fulfils for node $\boldsymbol{e}$ and reflects that node $\boldsymbol{e}$ is energy depletion node in the routing path then node $\boldsymbol{a}$ sends RERR message to the source node for a new path search.

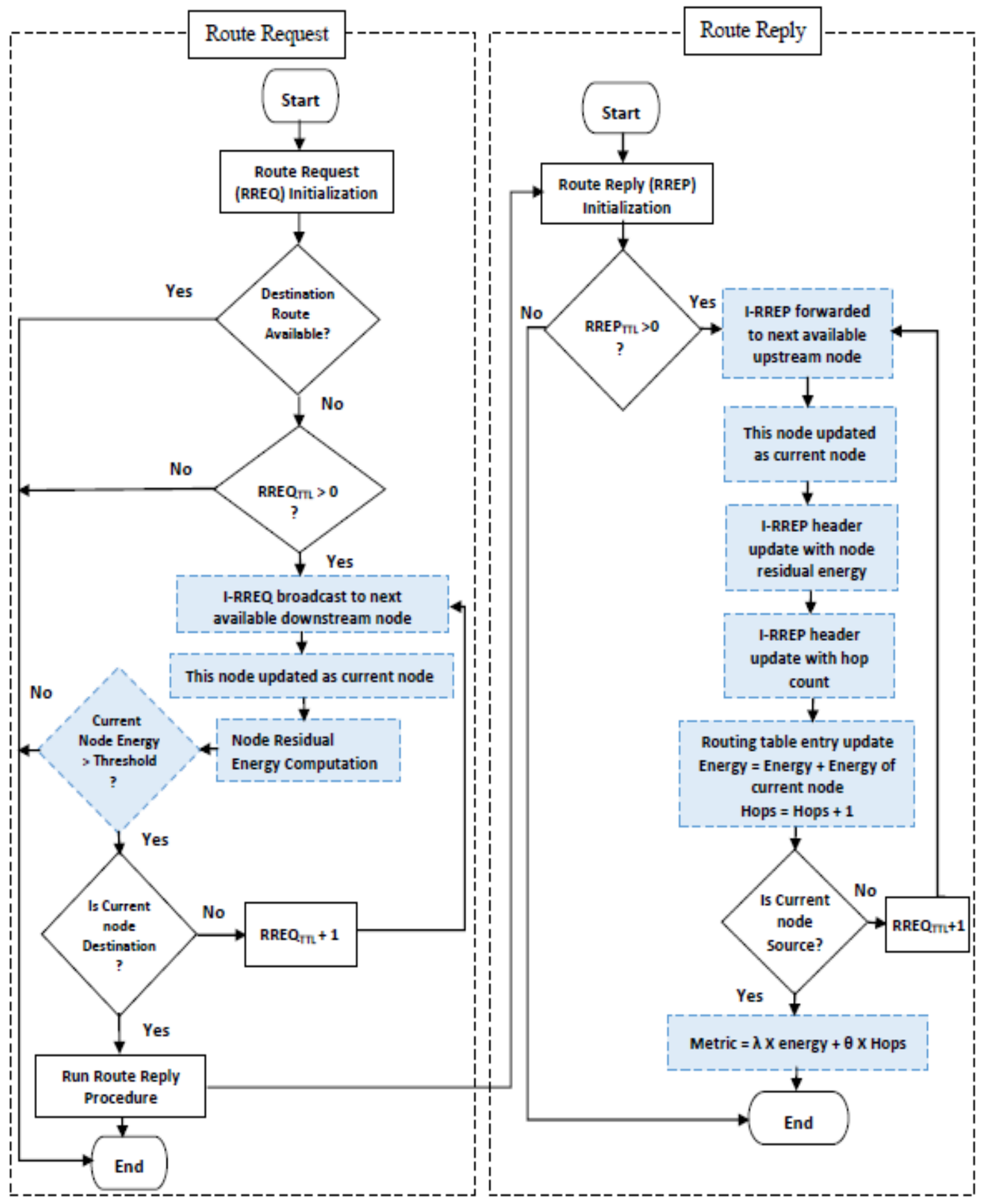

Figure 3. EARP route setup flow chart 


\section{PERFORMANCE EVALUATION}

NS-2 network simulator used in this study to evaluate the proposed EARP scheme performance in comparison with other researcher work. Randomly allocated 10 to 100 wireless nodes used in a Mobile Ad hoc Network for simulation environment. Random waypoint model and node mobility used in the network scenario. The flat area of 1200 by 1200 square meter considered for this simulation. The maximum speed of node is $10 \mathrm{~m} / \mathrm{s}$ whereas minimum speed is zero m/s. Pause time is 2 seconds while 512 bytes of data payload for traffic generation. The range for transmission of nodes is 250-meter power range. IEEE 802.11 considered for Medium Access Control. The node energy threshold value is fixed 50 joules out of 60 joules.

\section{RESULTS AND DISCUSSION}

Comparative results of network lifetime for EARP, ERB-AODV, M-AODV and AODV protocol represented in Figure 4(a). Average consumption of energy found to increase with the increment of network size. Therefore, it decreases network lifetime. However, proposed EARP scheme sustains relatively better performance of network lifetime as a result of selecting a route which has energy efficient nodes. It is necessary to avoid only node selection on the basis of the shortest path which drains those nodes energy quickly or nodes become selfish for saving their energy resources. Also, it is necessary to balance energy consumption between nodes by eliminating energy depletion node in route selection. Whereas, ERB-AODV, M-AODV and AODV protocol does not have any mechanism to check node remaining energy for balancing energy levels between nodes and uses those nodes in an active communication until end of their energy level. Therefore, it is clear from the simulation result in Figure 4 (b) that proposed EARP scheme performs better at packet delivery ratio in comparison with existing routing protocols.

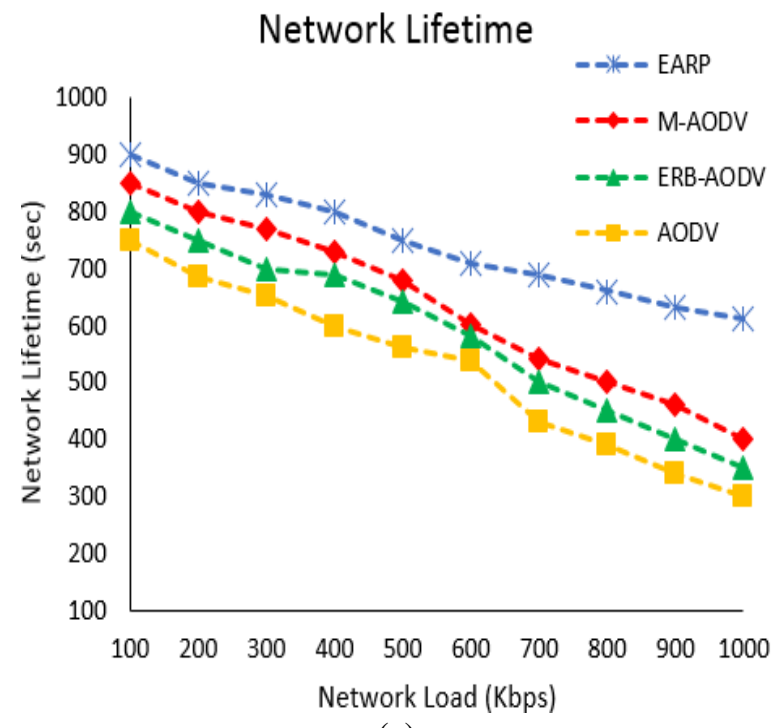

(a)

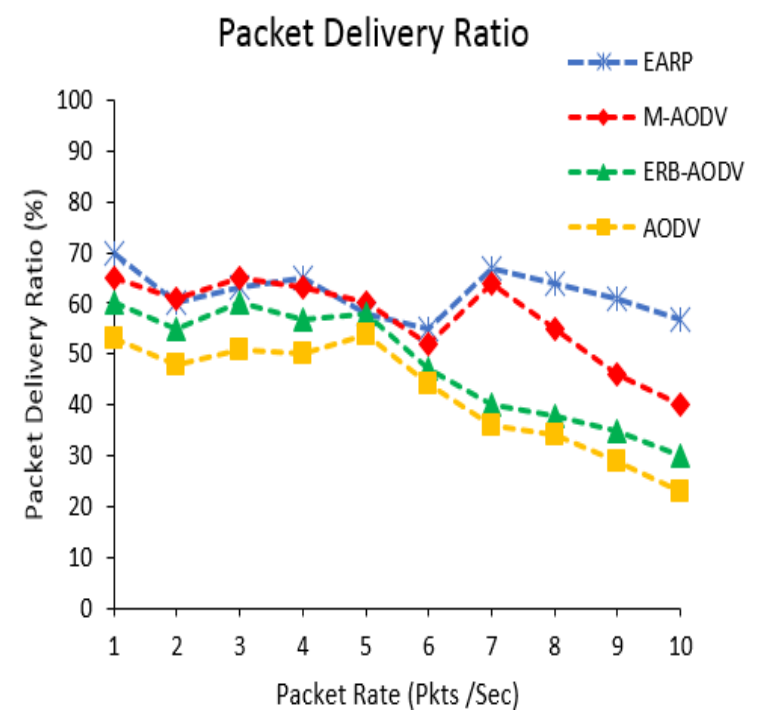

(b)

Figure 4. EARP performance comparison for network lifetime and packet delivery ratio

Simulation results for an end-to-end delay represented in Figure 5 (a). All the routing schemes show same performance at few nodes in the network due to less network interference. End-to-end delay increases remarkably with increment nodes number in the network, as a result of more congestion and network load. Existing ERB-AODV, M-AODV and AODV routing schemes use conventional route maintenance method which does not consider node energy in route selection process and consequently increase route instability. Whereas, EARP ensure that if there is an existence of energy deficient node in an active route it immediately sends early link failure report to the source node for broken link repair from energy efficient nodes and uses the shortest route from source to destination.

Subsequently, link breakage and frequency of route discovery minimized in EARP and end-to-end delay reduced. Figure 5 (b) shows the throughput performance of EARP scheme with variant node number in contrast with ERB-AODV, M-AODV and AODV routing schemes. All schemes show enhancement in performance with increasing nodes from 10 to 25 in the network due to load sharing for packet forwarding 
between nodes. However, more nodes are added up in the network, and when the number reached to 100, it found that as a consequence of incremental packet flood for the discovery of route and maintenance of route the network performance is decreasing. Whereas, in EARP relatively high throughput is maintained in more nodes in the network, as it enhances route selection and route maintenance while its on-demand nature makes it appropriate to adjust it with the variant network load. Moreover, for routing EARP use a composite metric which equally gives importance to hop count and node residual energy to limit the number of hops in shortest path selection.

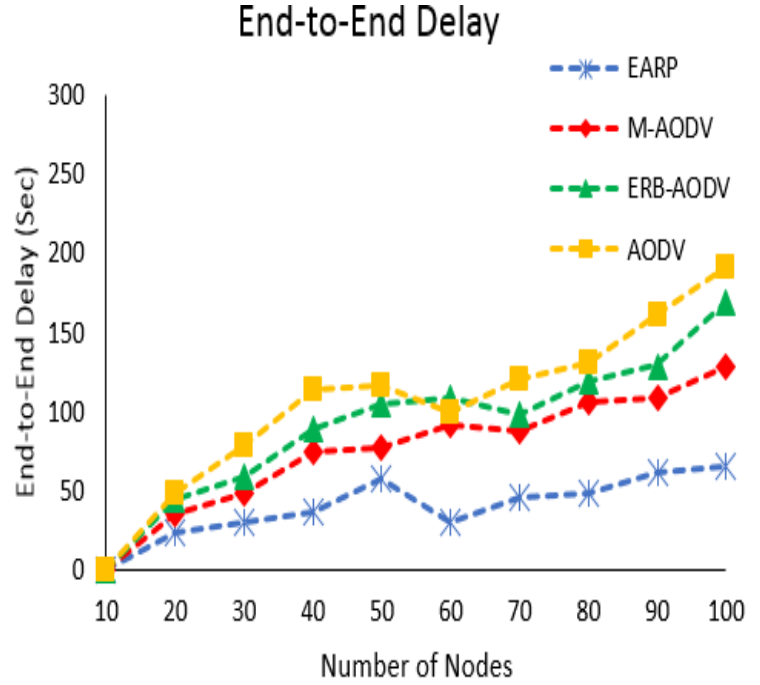

(a)

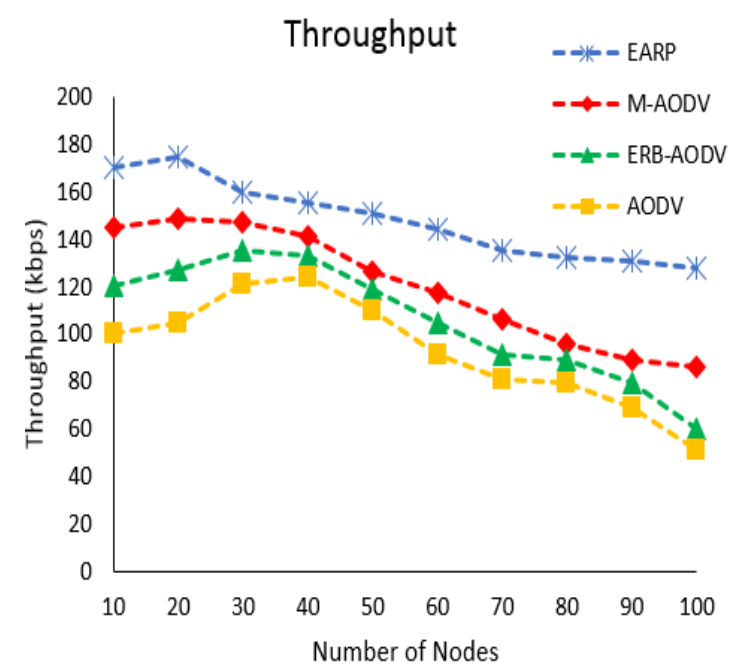

(b)

Figure 5. EARP performance comparison for end-to-end delay and throughput

Finally, proposed EARP scheme provides a multifaceted strategy for routing data packet by minimizing network overheads and overall routing for resource constraints mobile nodes. Comparative analysis of proposed scheme with other existing schemes represented in Table 2. EARP and M-AODV use composite routing metric according to network dynamic nature. In comparison with existing schemes proposed protocol experiences less routing and network load due to its straightforward and prosperous design without utilizing more broadcasts and interchange of control packets. Moreover, proposed protocol detects early link failure and repairs route with energy efficient nodes.

Table 2. Proposed Scheme Comparative Analysis

\begin{tabular}{lcccc}
\hline & EARP & ERB-AODV & M-AODV & AODV \\
\hline Routing Metric & Composite & Singular & Composite & Singular \\
Network Load & Low & Medium & High & High \\
Routing Overhead & Low & High & High & High \\
Link breakage Detection & Yes & No & Yes & No \\
\hline
\end{tabular}

\section{CONCLUSION}

In this paper, we recommended an easily deployable and lightweight Energy Aware Routing Protocol (EARP) for the mobile ad-hoc network to select energy efficient nodes in route selection and route repair. EARP can also distinguish a node with the low energy level in current routing path from source to destination. Therefore, it could repair broken link by selecting nodes with higher energy levels and chooses the shortest path. The efficiency of the proposed scheme proved by simulation results. Performance of EARP scheme compared with ERB-AODV, M-AODV and AODV routing protocol in the form of end-to-end delay, packet delivery ratio, network lifetime and throughput.

The results obtained from simulation show that overall performance of ERB-AODV, M-AODV and AODV is critically affected by energy depletion node which reduces the throughput and packet delivery ratio to extreme limits. Whereas, EARP significantly enhances the overall performance of the network by selecting 
an energy-efficient node and eliminating energy depletion node from route chosen before link breakage. In future, we plan to include link quality parameters in the selection of the route, therefore chosen routes to have less link loss ratio.

\section{REFERENCES}

[1] Havinal, R., G.V. Attimarad, and M. Giriprasad, EASR: Graph-based Framework for Energy Efficient Smart Routing in MANET using Availability Zones. International Journal of Electrical and Computer Engineering, 2015. 5(6).

[2] Hong, Y.S., A Control Packet Minimized Routing Protocol for Ad-hoc Wireless Networks. Indonesian Journal of Electrical Engineering and Computer Science, 2014. 12(2): p. 966-975.

[3] Djemili, F. and C. Tolba. A mixed approach load balancing and efficient energy for multi-path routing in mobile ad hoc networks. In 2013 IEEE SENSORS. 2013.

[4] Jamali, A., N. Naja, and D.E. Ouadghiri. An enhanced on ad hoc wireless network routing based on Aodv. In 2013 IFIP Wireless Days (WD). 2013.

[5] K, K., et al. Throughput enhancement by traffic splitting over an ad-hoc network with hybrid radio devices. In IEEE 2013 Tencon - Spring. 2013.

[6] Torrieri, D., S. Talarico, and M.C. Valenti, Performance Comparisons of Geographic Routing Protocols in Mobile Ad Hoc Networks. IEEE Transactions on Communications, 2015. 63(11): p. 4276-4286.

[7] Kumar, S.A., et al., An Empirical Critique of On-Demand Routing Protocols against Rushing Attack in MANET. International Journal of Electrical and Computer Engineering, 2015. 5(5).

[8] Costagliola, N., et al., Energy-and delay-efficient routing in mobile ad hoc networks. Mobile Networks and Applications, 2012. 17(2): p. 281-297.

[9] Ejmaa, A.M.E., et al., Neighbor-Based Dynamic Connectivity Factor Routing Protocol for Mobile Ad Hoc Network. IEEE Access, 2016. 4: p. 8053-8064.

[10] Singh, A. and D. Chadha, A Study on Energy Efficient Routing Protocols in MANETs with Effect on Selfish Behaviour. International Journal of Innovative Research in Computer and Communication Engineering, 2013. 1(7): p. 1386-1400.

[11] Shivashankar, G. Varaprasad, and S.H. Narayanagowda, Implementing a new power aware routing algorithm based on existing dynamic source routing protocol for mobile ad hoc networks. IET Networks, 2014. 3(2): p. 137-142.

[12] Paramasivan, B., M.J.V. Prakash, and M. Kaliappan, Development of a secure routing protocol using game theory model in mobile ad hoc networks. Journal of Communications and Networks, 2015. 17(1): p. 75-83.

[13] Shankar, S., G. Varaprasad, and H.N. Suresh, Importance of on-demand modified power aware dynamic source routing protocol in mobile ad-hoc networks. IET Microwaves, Antennas \& Propagation, 2014. 8(7): p. 459-464.

[14] Abbas, N.I., M. Ilkan, and E. Ozen, Fuzzy approach to improving route stability of the AODV routing protocol. Eurasip Journal on Wireless Communications and Networking, 2015.

[15] Djemili, F. and C. Tolba. A mixed approach load balancing and efficient energy for multi-path routing in mobile ad hoc networks. In IEEE SENSORS. 2013.

[16] Kuo, W.K. and S.H. Chu, Energy Efficiency Optimization for Mobile Ad Hoc Networks. IEEE Access, 2016. 4: p. 928-940.

[17] Bader, A. and M.S. Alouini, Mobile Ad Hoc Networks in Bandwidth-Demanding Mission-Critical Applications: Practical Implementation Insights. IEEE Access, 2017. 5: p. 891-910.

[18] Kumar, R., et al. An on demand routing protocol AODV with end to end reliability and backward route information. In IEEE 9th International Conference on Industrial and Information Systems (ICIIS). 2014.

[19] Zamani, E. and M. Soltanaghaei, The Improved Overhearing Backup AODV Protocol in MANET. Journal of Computer Networks and Communications, 2016.

[20] Jain, P. and A. Suryavanshi. Energy efficient Local Route Repair multicast AODV routing schemes in Wireless Ad hoc Network. In IEEE International Conference on Advanced Communications, Control and Computing Technologies. 2014.

[21] Abdulaziz Al-Nahari, Mohd. Murtadha Mohamad, and R. Zahilah, An Improved Maintenance Strategy In Ad Hoc On-Demand Routing Protocols For Mobile Ad Hoc Networks. Jurnal Teknologi, 2015.

[22] Nishanthini, C., G. Rajkumar, and G.N. Jayabhavani. Enhanced performance of AODV with power boosted alternate path. In IEEE International Conference on Computer Communication and Informatics. 2013.

[23] Liu, S., Y. Yang, and W. Wang, Research of AODV Routing Protocol for Ad Hoc Networks1. AASRI Procedia, 2013. 5: p. 21-31.

[24] Fadaly, H.E., R. Ramadan, and I. Talkhan. Residual Energy Considerations for AODV Enhancement. In IEEE 5th International Conference on Intelligent Systems, Modelling and Simulation. 2014.

[25] Mohapatra, S.K., et al. Stability and energy aware reverse AODV routing protocol in MANETS. In IEEE 2nd International Conference on Recent Trends in Information Systems (ReTIS). 2015. 